\title{
Swabbed Material
}

National Cancer Institute

\section{Source}

National Cancer Institute. Swabbed Material. NCI Thesaurus. Code C150895.

A heterogeneous and variable aggregate of body fluids, tissues, cells, microorganisms, and other materials that are collected from a swab biospecimen collection procedure. 